\title{
VICTIM EVACUATION TECHNIQUES IN EMERGENCY CONDITIONS
}

\author{
Paweł Gawlowski ${ }^{1}$, Alicja Biskup ${ }^{2}$ (1) \\ ${ }^{1}$ Department of Emergency Medical Service, Wroclaw Medical University, Poland \\ ${ }^{2}$ University Clinical Hospital in Wroclaw, Poland
}

\begin{abstract}
The victim at the scene of the incident very often requires evacuation from the danger area to the safe area or from the incident area to the hospital. The choice of technique depends on the number of rescuers available and the condition of the victim, with particular emphasis on serious and life-threatening injuries. Evacuation can take place without the use of equipment when the rescuer or rescuers carry the victim on their own hands. The optimal solution, especially for trauma patients, is to evacuate them using equipment that allows stabilization of the whole body and safe handling of the injured in vertical and horizontal planes.
\end{abstract}

KEY WORDS: evacuation; victim; medical rescue; rescue equipment; paramedic

Disaster Emerg Med J 2019; 4(3): 116-123

\section{INTRODUCTION}

Evacuation is an essential component of rescue services because efficient and fully executed proper protection of the victim can only be performed in a safe place where rescuers are not threatened by any hazards [1]. It is necessary to check if there is no danger of fire or explosives and other dangerous materials. Evacuation is essential if the rescuers are unable to protect the patient from other hazards, such as an attack from other persons, or if they cannot evacuate persons who require life-saving measures. The evacuation must also be carried out when it is not possible to aid a victim of a patient's location or position at a particular location when the necessary resources and facilities to save the life and health of another person are not available $[2,3]$.

In order for this to happen, it is first necessary to find the right way, so as not to cause any additional harm, to move the person to a place where all necessary medical rescue operations can be carried out. The way in which an injured person is evacuated or transported from one place to another depends on the in- jury suffered and the general condition of the person to be evacuated. Consider all possible options, such as cervical, thoracic, or lumbar spinal injury, pelvic fracture or injury, such as upper or lower limb bones injury, and consider the diseases or ailments the victim is complaining about, such as shortness of breath, chest pain, or other conditions requiring a different position for transport, or whether the woman being evacuated/transported is not visibly pregnant [4].

The right evacuation method is also important for the rescuers themselves, as a properly selected and properly executed method protects the rescuers from harm or personal injury, such as overload injuries [5]. The number of rescuers is also important, as some methods can be used by one and others require more people. This is related to the injuries suffered by the injured person, because more severely injured, usually traumatized, require greater stability and, for example, manual stabilization of the cervical spine, where one or even two people would not be able to cope with moving such a person on an orthopaedic board or even a stretcher. 
There are a few key principles to follow on the scene. First, do not move or relocate the injured person when this is not necessary. If you have access to a person who allows you to carry out all the necessary rescue operations and if the place is safe for you and there is no risk of the building collapsing, for example, you should do so in that position to prevent any secondary injury to the victim [6].

If the victim is conscious, inform them of all the actions taken by the rescuers and calm them down so as not to cause them concern and ensure their cooperation. His or her mental comfort is important because secondary injuries are not only bodily harm or disorderly functioning but also psychological trauma and associated trauma that affect the future and further life of the person [7].

To be able to work effectively with a victim, you must always be sure that the people you work with, as well as others who help with the move or other activities, follow our instructions and know what to do. If they do not know this, explain it to them or, if possible, show them when they are evacuating the victim.

When moving the victim, listen to the instructions given by one person. Then all tasks are performed simultaneously and at the right time because the person who gives the orders should usually be at the head of the rescued person and should observe his or her reactions to the team's actions [8]. This rescuer also has the widest field of vision when it comes to the position of the victim.

Follow the recommended rules and methods of patient handling to reduce secondary injuries and personal injuries, taking care of your own safety and the safety of the injured person.

\section{Non-instrumented means of evacuation of an injured person}

Conscious persons who are non-traumatic and stunned, for example by an explosion or a potentially dangerous event itself, or with injuries that allow them to move their lower limbs alone or with little or no assistance, are best simply to lead to a designated point within the danger zone. All in all, grasp the person's shoulder and allow him or her to support his or her weight and walk to the designated area as quickly as the condition of the evacuated person allows [9].

If, for some reason, the person is unable to move on his/her own - due to weakness or traumatic injuries in the lower limbs, or if he/she is unconscious and unable to follow our instructions - it is best to use the so-called "maternal method" to evacuate the person. Hold the arms of the person with one hand and put the other hand under his or her knees. This person can embrace the rescuer by the neck to support him/herself if he/she is conscious. If this is not possible, it does not significantly affect the technique and handling.

A conscious person who cannot walk alone can also be evacuated from a dangerous place by using the "ram" method. To do this, you need to squat, stand in front of the victim, take him/her with your forearms under your knees and thighs, and he/she embraces the rescuer behind the neck. Then the rescuer is lifted in balance and the victim is maintained.

The fire-fighting grip also allows for evacuation of both conscious and unconscious persons. It consists in capturing the victim and literally placing him on the shoulders of the rescuer. It is necessary to squat to catch the victim with one hand and put it between his legs and then put the rescued person on the shoulders of the rescuer. Hold the wrist of the hand hanging forward with the hand between the victim's legs. In this way, the injured can be carried even from a ladder, as one hand of the rescuer remains free. It can also be used to protect yourself and the injured person from, for example, tree branches or to support an object in another situation.

If there is only one rescuer who has to evacuate a person who cannot walk, is unconscious or conscious, but with injuries that do not allow the use of a mother's grip or a "ram" method, or is too heavy to use, from the danger zone, the rescuer can be dragged along the ground at close range [10]. Take your clothes or place a blanket underneath the evacuated person and pull on a relatively safe surface, flat and horizontal only, so as not to cause additional injury.

A lying person can also be evacuated from a dangerous place with a Rautek manoeuvre, which consists in kneeling down at the victim's head and placing hands under their shoulder blades so that the head rests on the lifeguard's forearms. Move gradually up to the victim and upwards, constantly supporting their body, head and back against their shoulders and chest to prevent secondary injuries. Put your hands under your armpits and grasp your forearm with both hands, one close to your wrist and the other close to your elbow. When making a squat, the rescuer must lift and support the victim's weight on his or her hip, and then straight- 
en and move backwards, pulling the person back to a safe place.

Sometimes there is a need to quickly evacuate a person involved in a car accident and to do so you need to use a special method that will allow you to take care of the condition and possible not to worsen the injuries of that person. Rautek manoeuvre is the safest way to pull an injured person out of a vehicle when they are in danger and have no one to help them. Place the head and chest of the rescuer on the rescuer's torso and put both hands under the shoulders of the injured person. Then the victim's forearm should be grasped with both hands, one closer to the wrist and the other closer to the elbow. The weight of the body to be pulled out should then be transferred to the rescuer and taken out of the vehicle. The victim's legs must have been previously freed from the seat and pedals so that they do not obstruct the pulling out of the car. This position, however, does not stabilize the cervical spine, so it can be modified by holding the jaw with one of the hands placed under the shoulder and pressing it against the rescuer's chest [2].

\section{Non-instrumented means of evacuation of an injured carried out by two or more rescuers}

Two rescuers can move an injured person by using the so-called "chair" method. The idea is that the rescuers, standing opposite each other, first grasp each of their own wrists with their hands and then braid their hands to grasp the other rescuer's free wrist. In this way, they create something like a square from their hands. In this position, they forge and the victim sits on their intertwined hands and forearms. It includes the necks of the rescuers, whom it has on both sides. They straighten up and can take the injured out of the room or place.

The "bench" way is very similar to the "chair" way. It is based on a different hand position. Two rescuers standing opposite each other stretch out their hands in front of each other and grab the wrists of the rescuer standing opposite, i.e. the left hand grabs the right wrist of the colleague and the right hand grabs the left. Each rescuer's forearms are arranged parallel to each other. The injured person can sit on both sides of the rescuers' joined forearms, but can also only sit on one side and be held by the rescuers' backs or arms by the rescuers.

To carry or evacuate a seated person quickly, you can use the fact that he or she is sitting on a chair. To evacuate a conscious person, it is enough to approach both shoulders of the victim and grasp the support of the chair below the seat. The rescuer can embrace the rescuers behind the neck for better stability and holding in this position, and the rescuers rise while lifting the chair up and out of the rescued person.

If the person is unconscious and is sitting on a chair and, for example, leaning on the table with his or her hands, hold the person constantly, tie the body to the chair in order not to cause him or her to slip out and fall off the chair. It is best to secure her with straps or even without straps to make her from clothes lying somewhere nearby. These belts should be tied up at the level of the victim's shoulders, pelvis and thighs. In this way, a secured person can be carried by lifting the chair slightly upwards by the rescuers, one to each side, or by pulling it down on the ground or in a slight lift, with one rescuer standing at the back and one at the front of the victim, and the chair leaning slightly backwards towards the rescuer at the back.

With three rescuers at your disposal, you can also carry the injured person on your hands. Then one supports the head, the other the shoulders, and the third leg of the injured person or one shoulder, the other the pelvis and the third the legs. The setting depends on the size of the person to be moved. If it is a child, one person will be able to support his head, because those two will secure the rest of his body. However, if it is an adult, it depends on the rescuers whether they will be able to lift the victim in one or the other way.

\section{Instrumental means of evacuation of the injured}

A patient without an injury is a person who is known not to have suffered an injury from a witness report, as well as from an interview with the victim himself, if he is conscious and trusted, and clinical examinations preclude him from suffering an injury. If the person is unconscious, it is only from the accounts of witnesses of the event that it can be assumed, after prior examination of the victim, that he did not suffer any injury.

When evacuating or transferring such a patient, care must be taken to maintain the airway of the patient's respiratory tract and to ensure his or her physiological position.

The most commonly used equipment is self-propelled stretchers on wheels. Whenever possible, they should be used to transport patients from the scene to the ambulance. They can only be used on level 
ground. They should be controlled by foot control. A rescuer standing on the head side pushes them with muscle strength [3]. When on uneven terrain, for example when descending stairs, it may sometimes be necessary to lift a stretcher on which the patient is already lying. The rescuers then stand one at the head and one at the feet and carry the victim along with the stretcher. The most appropriate and safe option would be to carry four people, as this allows you to control and maintain more balance and stability than two people.

The non-injured patient can be transferred to them in two ways: directly from the ground or using the technique of lifting the limbs. In order to move the victim directly from the ground, it is best to have three rescuers at your disposal. Then each one of them has a pony on one side of the rescuer. The rescuer who is closest to squat at head slides one hand under the victim's neck and shoulders to protect the head, and the other hand is placed under the upper part of the victim's chest. The next rescuer places his hands under the patient's waist and buttocks in turn. The third rescuer places both hands under the feet of the injured person. The victim's hands should be on the victim's chest.

If there are no three rescuers on-site, then two of them should cope with this task. If they are in an emergency situation where they are in danger, the two of them should move the patient. If not, they can wait for backup.

The first rescuer gives a signal to the others and all at the same time raise from their knees at the sign, lifting the rescued and slightly tilting him/her in his/her direction. The next signal is a sign to stand up and put the patient on a stretcher [11].

In turn, the technique of lifting the limbs requires only two rescuers to use it. One of them kneels at the head of the rescued person, and the other one at the side of his or her knees. The rescuer who is closer to the head puts his hands under the victim's shoulders and grabs his or her wrists, and the other one weaves his or her hands and places them under the rescuer's knees, trying to put his or her hands as close to his or her hips as possible to share the weight. The rescuers get up at the same time and put the patient on a stretcher.

In the same way, you can place the patient on a folding stretcher that can be folded in half when trying to get to a person who is in an inaccessible place where the stretcher on wheels will not fit. They are also used to transport another injured person in the same ambulance where the wheeled stretcher is located. There should be special seat belts in the patient compartment to protect the folding stretcher when the ambulance travels to the hospital.

Pick up stretchers are used to lift the victim in the back position and to transfer them to the transport stretcher. They have hinges that open at the head and feet of the person to be rescued, allowing them to be adjusted in length to suit the height of the patient. They should be placed next to the rescued person and the appropriate length should be measured. They should then be unfastened and placed under the victim on both sides to "pick him up" to close them around and under the patient. Do not transport the victim on a pick-up stretcher. They are only used to transfer to equipment suitable for evacuation and transport.

Soft stretchers are used without any damage to the patient's spine because they are made of a special material that is able to withstand their weight. They have handles on each corner that are used to carry the victim. They are ideal in situations where there is a need to lift a patient from stairs or on uneven terrain. They provide flexibility and durability.

Bucket stretcher is a rigid, safe device that can withstand very high loads in emergency situations. The victim is safe by remaining fastened inside the stretcher. Soft stretcher or pick-up stretcher or even a long orthopaedic board can be put into these stretchers. They can be tied to ropes or lifting equipment for carrying or lifting on uneven terrain.

The evacuation medical lift chair (cardiological patient chair) can be used to evacuate the patient downstairs or through narrow corridors. Two rescuers are sufficient to move the injured person by attaching them to the chair. Most chairs have wheels at the back legs so that the patient can be transported to the stretcher. Suitable for evacuating or transporting a person with shortness of breath.

It is important to adapt the way in which the injured person is transported to their condition of health [6], and therefore, paying attention to a woman in a visible pregnancy, she should be transported on her left side with her right hip elevated or preferably the whole right side of the body, because the woman's uterus lying on her back presses the aorta and the inferior vena cava, which limits the return of venous blood to the heart and reduces its ejection volume, causing a decline in blood pressure. This increases the venous pressure in the uterus, which can cause the placenta to detach. 
Patients with shortness of breath should be evacuated, if possible, in a sitting or half seated position as they have difficulty breathing when lying down and can activate additional respiratory muscles when sitting down to support breathing. Remember about the possibility of placing the patient flat in case of a deterioration of his condition and the need to undertake rescue procedures.

People with so-called acute abdomen should be placed on stretchers with bent legs in their knees to relax the abdominal muscles and alleviate pain. A rolled-up blanket can be placed underneath your knees or other material for this purpose.

\section{Instrumented means of evacuation of an injured}

If you are dealing with an unconscious patient and there are no witnesses on the scene, you should always suspect that the victim is a traumatized victim. Handle the patient with caution and limit the possibility of additional traumatization by securing the spine in each section of the spine, especially in the cervical section. The final immobilization is correct when the patient is attached to a stretcher or board with at least three straps and an orthopaedic collar and the head is stabilized by the lateral head stabilizers (Fig. 1 and 2). If there are not enough rescuers on-site to evacuate the patient safely, and the scene is dangerous for the injured or the patient's condition requires immediate evacuation, the patient should be removed as a priority, saving his life. If the victim is stable and there is no danger at the scene, the cervical section of the spine is securitized, and the necessary rescue operations are performed while waiting for backup [12].

There are several methods of carrying a victim on a stretcher, which depends on the number of

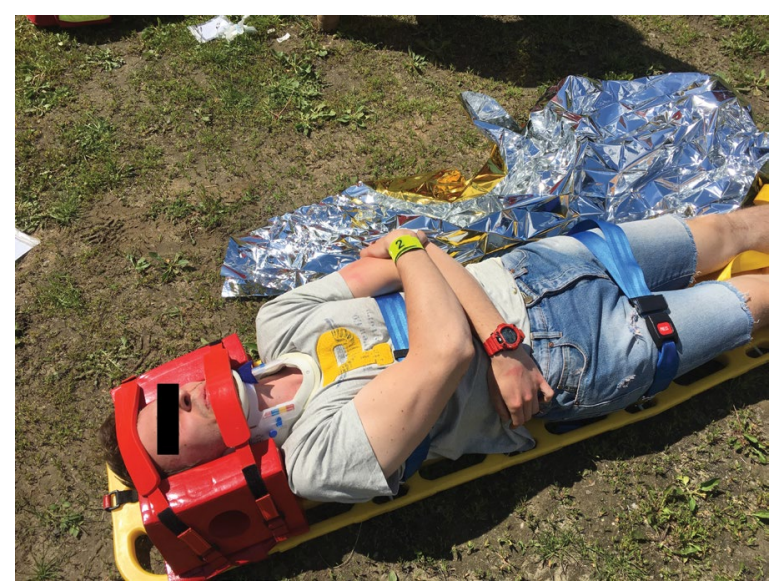

FIGURE 1. Trauma patient secured on the board during medical segregation at the scene of the incident rescuers or people who will perform this task [13]. The most atraumatic method can be used when five people are available. They do not have to be paramedics or even rescuers, but if the medical services cooperate with other people, they have to explain everything to such people so as not to cause secondary injuries to the patient. However, it is best to do this with qualified rescuers, but not always there are five of them on the spot. When carrying an injured person in such a way, it is important to remember to protect his cervical spine [14]. One of the rescuers is responsible for the head and its protection, while the rest of the rescuers line up two on each side of the injured person's shoulders and hips. Slide your hands as deeply as possible under the victim's chest, hips, thighs and thighs, and at the appropriate sign of the person securing the injured person's head, lift the rescued upwards. Then it can be transferred to the stretcher that has been placed or prepared [4]. These stretchers can be a vacuum mattress or an orthopaedic board, which are best suited to stabilize the trauma patient. The orthopaedic board is a hard and inconvenient device for the patient when used for a longer period of time. It is designed to significantly improve the quality of rescue operations performed by rescuers - from the place of action, through transport, to hospital treatment, while the vacuum stretcher on which the patient is placed, and then pumped to perfectly fit the body of the victim, are more comfortable and through their adaptation do not cause secondary injuries to the victim, caused by a long time lying in one position.

When four rescuers move a patient onto a stretcher, one of them is still responsible for securing his cervical spine. The stretcher is then placed

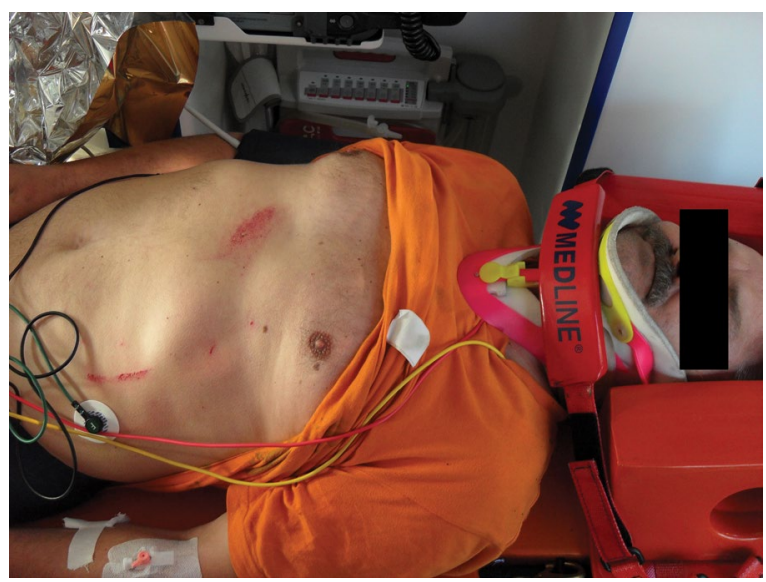

FIGURE 2. Patient with chest injury secured on the orthopaedic board during transport to the hospital 
next to the injured person and the rescuers stand astride the injured person and the stretcher $[15,16]$. They have to bend their legs in their knees and bend down to hold the injured person by the cage, hips and thighs with both hands, and at the signal given by the head protection person, lift the patient up and place the injured person on the stretcher. In this case, the orthopaedic board works best, because it can be laid sideways to the victim and when lifted up, it will fall to the ground in the place where the injured person was already lying, so you only need to put him back in the same place.

Trauma patients can also be transferred to stretchers in three persons, but this method is not suitable for victims with the unstable pelvis, as it does not provide adequate stability and immobilization during transfer. Still, one of the rescuers protects the victim's head and cervical spine [17]. Two of them kneel on one side of the injured person and grasp the rest of the patient's body alternately, placing their hands on the shoulder, pelvis, thighs and shin. The sign rotates sideways towards the rescuers. Right next to the sign, a previously prepared orthopaedic board is laid and the injured person is turned back on his back. At least three belts and stabilizers, i.e. blocks to immobilize the victim's head, should be used for the board.

Only pick-up stretchers may be used by two persons to lift the victim. One person keeps the head and cervical spine stable at all times, even when the patient is wearing an orthopaedic collar. The second person prepares the stretchers by selecting their appropriate length, unbuttoning them and placing them on both sides of the victim. Then he fastens the lock from the stretcher on the head side, and then from the legs side. The rescued can only be carried to the proper transport stretcher, which can be a vacuum mattress or an orthopaedic board. If the pick-up stretchers are placed on a stretcher in an ambulance, it can lead to spinal damage. With pick-up stretchers, pay attention to the different arrangement of the side stabilizers than with the orthopaedic board.

A person sitting in a car can be safely evacuated by two rescuers. This should be done with a Kendrick device (KED) [18]. If properly installed, it is sufficient to immobilize the head when an orthopaedic collar is also used $[19,20]$, as well as the chest and pelvis of the injured person. It has several belts that are fastened in the right order: first the middle chest belt, lower chest belt, hip belts, then head stabilizing belts, and finally the upper chest belt. One of the rescuers puts the orthopaedic board under the rescue's buttocks, resting one of its ends on the seat, and the other rests it on its own bent thighs. The second rescuer secures the position of the victim's legs and his partner pulls the patient onto the board by means of axial traction. After repositioning on the board, loosen the hip belts, straighten the victim's limbs and also loosen the chest belts. Fasten the patient to the board with three straps and attach the head stabilizers and remove the injured person from the car [21]. The KED is used for stable patients who have no significant cardiopulmonary disorders [22].

The two rescuers can also place the victim on the orthopaedic board from a standing position. The board should be placed at the patient's back and the rescuers face the patient on both sides [23]. Supporting the board with your feet, each of them puts his or her hand closer to the victim under his or her arm and grabs the board at one level. The other hand can be used to secure the cervical spine of the evacuated person. Slowly walking, lower the board slowly and place the injured person on it.

The so-called Pedi-Pack stretcher is prepared and adapted for the evacuation of paediatric injured patients. It is a sort of orthopaedic board, which is adapted for children. You can also use a small vacuum mattress for this. You can also use the KED vest in an emergency, in a standard form or inverted upside down.

\section{SUMMARY}

There are many ways of evacuating and transporting an injured person, depending on the injury suffered and the terrain in which they are located. If it is a difficult mountainous area, different methods will be used than if the victim is in the water.

In different cases, the methods of proceeding have been defined according to the given area, but sometimes they need modification by rescuers because each of these situations is different, the weather conditions and the terrain are also different. The equipment is diverse and prepared for different possibilities, but it will not always meet the expectations and requirements of the people using it.

The rescuer should first of all take care of his own safety because it is the key to saving someone's life. If the rescuer will not be able to help anyone, and additionally will need help from outside, it may 
cause loss of life and health. In difficult terrain, if it is not possible to save all the victims, it is necessary to save those who can be saved and not expose oneself to danger.

If the site is safe and therefore the rescuers are not threatened by any danger, there is time to think about and choose the best method of transport from the area for the victim. Then the rescuers have time for any medical interventions. However, if this is an area threatened by explosive substances, fire or terrorist attack, it is only necessary to secure the victim and as soon as possible move away with him to such a place that will be safe for him and the rescuer. Only then should the patient's injuries or illnesses be dealt with.

It is important to note that patients may need different forms of support, ranging from psychological support and recovery from difficult terrain to real life-threatening at a given time. Dealing with a conscious and informed person is different, and many different dealings with an unconscious person who has a multi-organ trauma and must be specialized in order not to aggravate the injuries.

It is important to choose the most convenient means of evacuation and transport for both the patient and the rescuers. Note that different terrain does not mean much more varied equipment. It is only more suited to the prevailing conditions, whether it is snow or water.

A vacuum mattress is the most suitable equipment for an injured patient who needs to stabilize the whole body in one position. And this position must not cause discomfort to the person as it can cause secondary injury. It is used on land, snow or in the air, so it is widely used.

Rescuers should not complicate their work and use methods that are as effective as possible. If more people are available, you can ask someone for help to help you and the victim you need to move. Pay attention to your surroundings and know how to evacuate the patient in order to make work easier for yourself and thus relieve some of the patient's pain and suffering.

\section{REFERENCES}

1. Brown J, Sajankila N, Claridge JA. Prehospital Assessment of Trauma. Surg Clin North Am. 2017; 97(5): 961-983, doi: 10.1016/j. suc.2017.06.007, indexed in Pubmed: 28958367.

2. Kool B, Lilley R, Davie G. Prehospital injury deaths: The missing link. Injury. 2017; 48(5): 973-974, doi: 10.1016/j.injury.2017.03.001, indexed in Pubmed: 28283182.
3. Tobey N, Waseem ME. Chest Injury. StatPearls [Internet]. Treasure Island (FL): StatPearls Publishing. 2019.

4. Atiksawedparit $P$, Rattanasiri $S$, Sittichanbuncha $Y$, et al. Prehospital prediction of severe injury in road traffic injuries: A multicenter cross-sectional study. Injury. 2019 [Epub ahead of print], doi: 10.1016/j.injury.2019.05.028, indexed in Pubmed: 31174870.

5. Pélieu I, Kull C, Walder B. Prehospital and Emergency Care in Adult Patients with Acute Traumatic Brain Injury. Med Sci (Basel). 2019; 7(1), doi: 10.3390/medsci7010012, indexed in Pubmed: 30669658.

6. Koser BW, Suchenski ME. Evacuation Triage. StatPearls [Internet]. Treasure Island (FL): StatPearls Publishing. 2019.

7. Johari HG, Eskandari S. New trauma protocols versus advanced trauma life support recommendations. J Trauma. 2011; 71(4): 1097, doi: 10.1097/TA.0b013e3182302847, indexed in Pubmed: 21986764.

8. Kool DR, Blickman JG. Advanced Trauma Life Support. ABCDE from a radiological point of view. Emerg Radiol. 2007; 14(3): 135-141, doi: 10.1007/s10140-007-0633-x, indexed in Pubmed: 17564732.

9. Hoyt DB. Looking forward. Advanced trauma life support course. Bull Am Coll Surg. 2013; 98(8): 8-10, indexed in Pubmed: 24205569.

10. Bazyar J, Farrokhi M, Khankeh H. Triage Systems in Mass Casualty Incidents and Disasters: A Review Study with A Worldwide Approach. Open Access Maced J Med Sci. 2019; 7(3): 482-494, doi: 10.3889/ oamjms.2019.119, indexed in Pubmed: 30834023.

11. Gold CR. Prehospital advanced life support vs "scoop and run" in trauma management. Ann Emerg Med. 1987; 16(7): 797-801, doi: 10.1016/s0196-0644(87)80578-4, indexed in Pubmed: 3296877.

12. Werneburg I, Wilson $L A B$, Parr WCH, et al. Evolution of neck vertebral shape and neck retraction at the transition to modern turtles: an integrated geometric morphometric approach. Syst Biol. 2015; 64(2): 187-204, doi: 10.1093/sysbio/syu072, indexed in Pubmed: 25305281.

13. Wojewodzka-Zelezniakowicz M, Madziala A, Madziala M. Comparison of the Miller and Macintosh laryngoscopes in simulated pediatric trauma patient: a pilot study. Disaster and Emergency Medicine Journal. 2017; 2(1): 1-6, doi: 10.5603/demj.2017.0001.

14. Gawlowski P, Pyda S, Lukawski K, et al. Medical emergency and battlefield medicine. Disaster and Emergency Medicine Journal. 2019; 4(1): 18-28, doi: 10.5603/demj.2019.0005.

15. Wiles MD. ATLS: Archaic Trauma Life Support? Anaesthesia. 2015; 70(8): 893-897, doi: 10.1111/anae.13166, indexed in Pubmed: 26152249.

16. Yakubtsevich $R$, Dunder $D$, Kranc $K$, et al. Place of cervical collar in emergency medicine. Disaster and Emergency Medicine Journal. 2018; 3(3): 108-109, doi: 10.5603/demj.2018.0024.

17. Galvagno SM, Nahmias JT, Young DA. Advanced Trauma Life Support Update 2019: Management and Applications for Adults and Special Populations. Anesthesiol Clin. 2019; 37(1): 13-32, doi: 10.1016/j. anclin.2018.09.009, indexed in Pubmed: 30711226.

18. Markenson D, Foltin G, Tunik M, et al. The Kendrick extrication device used for pediatric spinal immobilization. Prehosp Emerg Care. 1999; 3(1): 66-69, indexed in Pubmed: 9921744. 
19. Misasi A, Ward JG, Dong F, et al. Prehospital Extrication Techniques: Neurological Outcomes Associated with the Rapid Extrication Method and the Kendrick Extrication Device. Am Surg. 2018; 84(2): 248-253, indexed in Pubmed: 29580354.

20. Bucher J, Dos Santos F, Frazier D, et al. Rapid Extrication versus the Kendrick Extrication Device (KED): Comparison of Techniques Used After Motor Vehicle Collisions. West J Emerg Med. 2015; 16(3): 453-458, doi: 10.5811/westjem.2015.1.21851, indexed in Pubmed: 25987929.
21. Reynard FA, Flaris AN, Simms ER, et al. Kendrick's extrication device and unstable pelvic fractures: Should a trochanteric belt be added? A cadaveric study. Injury. 2016; 47(3): 711-716, doi: 10.1016/j. injury.2016.01.028, indexed in Pubmed: 26867981.

22. Cayten CG, Murphy JG, Stahl WM. Basic life support versus advanced life support for injured patients with an injury severity score of 10 or more. J Trauma. 1993; 35(3): 460-6; discussion 466, doi: 10.1097/00005373-199309000-00021, indexed in Pubmed: 8371307. 